\title{
«Archivi delle emozioni»
}

\section{Una presentazione}

\author{
di Gabriele Scaramuzza
}

La rivista, dal titolo quanto mai stimolante "Archivi delle emozioni», reca a sottotitolo «Ricerche sulle componenti emotive nella letteratura, nell'arte, nella cultura materiale» ${ }^{1}$. È stata fondata ed è diretta da Sotera Fornaro, che in ogni numero antepone un editoriale proprio, indispensabile a fornire proposte di senso utili a orientarsi negli intrichi delle tematiche affrontate. Ancorché esperta, e docente, di vari aspetti dell'antichità classica, greca in particolare, Fornaro ha una grande attenzione per la comparazione tra le letterature, le culture anzi, di volta in volta in causa. Questo traspare chiaramente già nei modi in cui svolge i problemi, nell'impostazione di "Archivi delle emozioni». Che non è una rivista di storia della cultura, ma "di cultura" certamente sì; come a mio avviso il termine stesso "archivi" suggerisce.

Colpisce la spregiudicatezza nell'accostamento degli autori e dei tempi e degli spazi culturali cui appartengono. Tempi e spazi che contestualizzazioni a sfondo storicistico assegnano a distanze inconfrontabili; ma che vengono qui accostati, e dunque posti in relazione. L'antistoricismo (come l'antipsicologismo e l'antinaturalismo) della fenomenologia delle origini, alla base delle intenzioni che animano «Materiali di Estetica», sono evidenti anche qui.

Così il primo numero, uscito nel gennaio del 2020, è dedicato a La vergogna e le sue maschere. Il tema della vergogna (che torna anche nei numeri successivi) è fondamentale nella nostra cultura: basti pensare al rilievo che

\footnotetext{
1 «Archivi delle emozioni. Ricerche sulle componenti emotive nella letteratura, nell'arte, nella cultura materiale» [on-line]. Disponibile all'indirizzo: https://archivi-emozioni.it/index.php lrivista.
} 
assume nel Processo di Kafka, a Vergogna di Coetzee, alle riflessioni sul mondo concentrazionario, e (per stare al mondo culturale che ci è più prossimo) al recente Sentimenti di prorompente vergogna di Andrea Bonomi.

Il tema è affrontato per nuclei di emergenza appartenenti a epoche e a luoghi culturali differenti: all'Editoriale di Sotera Fornaro seguono in questo caso (esemplifico, come poi sempre, senza alcuna velleità di completezza) saggi relativi alla Francia postrivoluzionaria; a Charles Dickens, alla cultura americana; a Philip Roth, a Dostoevskij; a Michail Bulgakov; a Elfriede Jelinek; infine gli scrittori dell'esilio tra XX e XXI secolo.

Il secondo numero (del settembre 2020) ha per oggetto: Ri/sentimenti. Emozioni e linguaggi. E contiene, oltre al consueto editoriale di Sotera Fornaro, saggi sulla quinta ode di Bacchilide, sull'arte dell'improvvisazione in giocolieri, prestigiatori e oratori; sulla poesia del Rinascimento, sul linguaggio degli affetti in Dido di Johann Elias Schlegel, su empatia e spaesamento.

L'ultimo numero, uscito nel luglio del 2021, ha per titolo Sconfinamenti emotivi. Dalla tragedia greca all'intelligenza artificiale. Già il titolo dell'Editoriale ne prospetta i motivi di fondo: "Le emozioni dei robot, l'arte, la letteratura. Qualche considerazione”. Seguono saggi sulla tragedia greca, sul Filottete in particolare, sugli Argonauti di Flacco; su Ovidio; su "Scorci contemporanei sul dolore: sconfinamenti tra letteratura e cura. Infine su Marguerite Duras e su Annie Ernaux.

C'entra l'estetica in tutto questo? Certamente sì, se non si limita l'estetica ad ambiti inaccettabilmente ristretti. Come «Materiali di Estetica» si propone di fare; ricorda la presentazione a essa, che vale anche per l'estetica quanto si è affermato per la filosofia: «è un livello di metariflessione il cui oggetto si sposta continuamente». Di simili facoltà metariflessive non manca "Archivi delle emozioni». 